\title{
PREVALENCIA Y SINTOMATOLOGÍA DEL ESTRÉS POST TRAUMÁTICO EN PERSONAS QUE EXPERIMENTAN UN TERREMOTO Y UN TSUNAMI
}

PREVALENCE AND POST-TRAUMATIC STRESS SYMPTOMS IN PEOPLE WHO EXPERIENCE AN EARTHQUAKE AND TSUNAMI

Recibido: 27 de Mayo del 2013 | Aceptado: 13 de Julio del 2013

\author{
MARCELO LEIVA-BIANCHI ${ }_{1}$; ANDREA ARANEDA 2
}

(UNIVERSIDAD DE TALCA, Talca, Chile)

\begin{abstract}
RESUMEN
Introducción: Se sabe que la prevalencia del estrés post-traumático (TEPT) es significativamente mayor después de terremotos y tsunamis como el del 27 de febrero de 2010 (27-F). Objetivos: (1) Determinar las proporciones de personas que podrían presentar TEPT y cada uno de sus tipos de síntomas, (2) relacionar las proporciones con grupos de variables que pueden favorecer su aparición, y (3) establecer un perfil de síntomas según cada grupo de variables. Método: Mediante las escalas Short Posttraumatic Stress Disorder Rating Interview (SPRINT-E) y la Davidson Trauma Scale (DTS), medimos la cantidad de síntomas intensos de TEPT en dos muestras: habitantes del litoral $(n=194)$ y del interior $(n=94)$ de la zona central de Chile. Resultados: La prevalencia del TEPT alcanza el 22\%, siendo 19 puntos mayor en personas que habitan la zona costera (28\%) y 11 puntos mayor entre quienes han sufrido graves daños en su vivienda. Discusión: Quienes sufren graves daños 0 viven en una ciudad costera impactada por terremoto y tsunami tienden a tener más síntomas de re-experimentación e híper-activación, se muestran más tristes, tienen menos cuidado por su salud, se sienten incómodos e interferidos en sus actividades cotidianas y se les hace difícil resolver problemas que antes solucionaban sin ayuda.
\end{abstract}

PALABRAS CLAVE: Trastorno por Estrés Postraumático, Prevalencia, Terremotos, Tsunamis (fuente: MeSH, NLM).

\section{ABSTRACT}

Introduction: Previous research indicates an increased prevalence of post-traumatic stress disorder (PTSD) after earthquakes and tsunamis as the February 27, 2010 (F-27). Objectives: (1) To determine the proportions of people who could present PTSD and each of its types of symptoms, (2) relate the proportions with groups of variables that may favor its appearance, and (3) establish a symptom profile as each group of variables. Method: Using the Short Posttraumatic Stress Disorder Rating Interview (SPRINT-E) and the Davidson Trauma Scale (DTS), we measured the PISD intense symptoms in two samples: inhabitants of coast $(n=194)$ and interior $(n=94)$. Results: The PTSD prevalence was $22 \%, 19$ points higher in people that live in coastal area $(28 \%)$ and 11 points higher among people that suffered severe damage in their home. Discussion: Those who suffer serious house damages or live in a coastal town hit by earthquake and tsunami tend to have more symptoms of re-experiencing, hyper-activation, are sadder, have less care for their health, feel uncomfortable and interfered in their daily activities and they find difficult to solve problems that previously solved without help.

KEY WORDS: Post-Traumatic Stress, Prevalence, Earthquakes, Tsunamis (source: MeSH, NLM). 


\section{INTRODUCCIÓN}

El 27 de febrero de 2010 (27-F), a las 03:34 hora continental chilena, se produjo el sexto terremoto más intenso ( $8.8^{\circ}$ Richter) desde que existen registros fiables. Presentó una duración de 3 minutos aproximados y su epicentro $\left(35,9^{\circ}\right.$ latitud sur; $72,7^{\circ}$ longitud oeste) a 335 kilómetros al suroeste de la capital Santiago de Chile, lo ubicó en una zona altamente poblada (USGS-United States Geological Survey, 2013). Luego del terremoto, se produjo un tsunami que destruyó las costas de la zona central especialmente de la Región del Maule. En esta región, ambos eventos causaron 320 víctimas fatales y 86.233 damnificados. En el caso de Constitución, la principal ciudad costera del Maule, tres olas de 9 metros, ingresaron por la desembocadura del río Maule, devastando gran parte de la ciudad. Se transformó así en la ciudad más afectada por el 27-F (PAHO, 2010).

Terremotos y tsunamis son eventos de alto impacto psicológico y social para la población afectada. Su más importante consecuencia en la salud mental de la población es el aumento de los síntomas de estrés post-traumático (TEPT; Rodriguez, Zaccarelli, \& Perez, 2006; Solvason, Ernst, \& Roth, 2003). El TEPT se traduce en recuerdos y sueños estresantes, recurrentes e involuntarios, y reacciones disociativas, que van desde imágenes intrusivas hasta la sensación de que se está viviendo nuevamente el evento (APA, 2005; Friedman, Resick, Bryant, \& Brewin, 2011; Miller et al., 2012). Esto produce malestar psicológico y alta activación, dificultades para dormir, irritabilidad, desconcentración y sobresalto. Todas estas alteraciones se producen al menos después de un mes de ocurrido el evento y provocan deterioro social, laboral o de otras áreas importantes de la persona (APA, 2005). La literatura es heterogénea respecto de la prevalencia del TEPT después de un terremoto, fluctuando entre un $4 \%$ y un $42 \%$ (Bland et al., 2005; Cairo, Dutta, \& Nawaz, 2010; Dewaraja \&
Kawamura, 2006; Lai, Chang, Connor, Lee, \& Davidson, 2004; McMillen, North, \& Smith, 2000; Roussos et al., 2005; Sharan, Chaudhary, Kavathekar, \& Saxena, 1996; $\mathrm{Wu}$ et al., 2006). Antes del 27-F esta prevalencia era de $4,4 \%$ en la población general (Pérez et al., 2009), mientras que seis meses después del 27-F llegó al 12\% (Larrañaga \& Herrera, 2010).

Sin embargo, después de un desastre como el 27-F, no todas las personas sufren síntomas de TEPT. La depresión también ha sido reportada como una reacción psicológica a un desastre (Bödvarsdóttir \& Elklit, 2004), reacción que es comórbida con el TEPT (Campbell et al., 2007; Canetti et al., 2010; Davidson, Hughes, Blazer \& George, 1991; Eksy et al., 2007; Goenjian et al., 2000; Goenjian et al., 2008) y cuya prevalencia puede llegar al $45,8 \%$ después de un tsunami (Dewaraja \& Kawamura, 2006). Por otro lado, aunque algunas personas presentan esos trastornos, Norris, Friedman y Watson (2002) encontraron que, más a menudo, un desastre es seguido por reacciones psicológicas no específicas de angustia y diferentes problemas de salud en la población afectada. Después del terremoto ocurrido en Hanshin Awaji en 1995, el TEPT fue una parte de la variedad de problemas de salud mental que presentaron los sobrevivientes, por ejemplo ansiedad, problemas del sueño, depresión y abuso del alcohol (Shinfuku, 2002). En el contexto latinoamericano, un $83 \%$ de los sobrevivientes mexicanos a desastres reportan síntomas que no pueden ser clasificados específicamente como TEPT, por ejemplo "ataques de nervios", depresión y quejas somáticas (Norris, Weisshaar, Conrad, Diaz, Murphy, \& Ibañez, 2001). Con base en evidencia como la anterior, Hamblen y sus colaboradores (2009) acuñaron el término de "postdisaster distress" o estrés post-desastre (EPD). El EPD reúne una serie de reacciones de tipo emocional, cognitivo y conductual frente a un desastre, incluyendo síntomas de TEPT, 
depresión, vulnerabilidad al estrés y dificultades en el funcionamiento.

Dado el contexto anterior, en este artículo determinaremos las siguientes proporciones: (1) personas que presentan TEPT y EPD; (2) personas que presentan cada uno de los síntomas y reacciones correspondientes al TEPT y EPD; y (3) personas que, agrupadas según algunas variables socio-demográficas y de exposición (p.e. haber o no sido expuesto al evento doble terremoto y tsunami), presentan TEPT, EPD y cada uno de sus síntomas. Finalmente, daremos un perfil de síntomas según cada grupo de variables que podría ser útil para identificar casos en riesgo de salud mental ante inminentes futuros desastres como el 27-F.

\section{MÉTODO}

\section{Participantes}

Participaron 288 personas seleccionadas de manera intencionada y cuya edad promedio fue de 35 años (Min=18; Max=65, DT=11). 194 pertenecen a la ciudad de Constitución y 94 a dos ciudades del interior de la zona central (Talca y Santiago). Dividida por sexos, las muestras se componen de 73 hombres (25\%) y 215 mujeres (75\%). Respecto de la ayuda estatal recibida después del evento, 77 personas declararon haberla recibido $(27 \%)$ y 211 no $(73 \%) .221$ personas declararon haber sufrido daños leves en su vivienda (sin daños o solo grietas; $77 \%$ ), mientras que 67 sufrieron daños severos (caída de muros/techos 0 pérdida total; 23\%). Finalmente, 138 personas no perdieron enseres (48\%) y 150 sí (52\%). Además, los participantes decidieron voluntariamente contestar las escalas, asegurándoseles el uso confidencial de la información mediante un consentimiento informado que debian previamente leer y firmar. Todos los participantes pertenecian a alguna organización (centro de salud, servicio público, profesores y apoderados de un colegio, estudiantes universitarios) y fueron encuestados de manera directa en su respectivo contexto organizacional.

\section{Instrumentos}

Para evaluar la probabilidad de que los participantes presentasen TEPT utilizamos dos escalas: Short Post-traumatic Stress Disorder Rating Interview (SPRINT-E) y Davidson Trauma Scale (DTS). SPRINT-E posee 12 preguntas que detectan síntomas de TEPT y estrés post-desastre (EPD; Hamblen et al., 2009; Norris \& Elrod, 2006; Norris, Hamblen, Brown, \& Schinka, 2008). La escala mide síntomas de reexperimentación (RE), evitación (EV), embotamiento (EM), híper-activación (HA), depresión (D), comportamientos poco saludables (CS), tolerancia al estrés (TE), mal desempeño en actividades diarias (DT), mal funcionamiento social (FS), preocupación por las propias reacciones (PR), necesidad de ayuda (NA) e ideación suicida (IS; Norris et al., 2008). Cada pregunta posee una escala de intensidad de 0 (mínima) a 4 (máxima), excepto el ítem 12 que es dicotómico ( 0 "sin ideación" y 1 "con ideación"). Si la respuesta a una pregunta es mayor o igual a 3 puntos (1 para el ítem 12), se considera como intensa. Un total de 30 más respuestas intensas indican una alta probabilidad de TEPT (Norris et al., 2008). SPRINT-E posee excelentes indicadores de fiabilidad $(a=, 916)$ y validez (CMIN $=2,237, \quad R M S E A=, 092, \quad N F I=, 901$, $\mathrm{CFI}=, 942$ y $P N F I=, 704)$ en la población a la que esta muestra pertenece (Leiva-Bianchi \& Gallardo, 2013).

Por su parte DTS está compuesta por 17 ítems agrupados según los criterios RE, EV/EM y HA. Cada ítem, es evaluado desde dos perspectivas, ambas en una escala de 0 (nunca/nada) a 4 (a diario/extrema) puntos: una para la frecuencia (cantidad de veces que le ha sucedido) y otra para la intensidad (magnitud o gravedad) con la que ha experimentado el síntoma. Se considera como límite para determinar si alguien 
presenta TEPT una puntuación total mayor o igual a 40 (Bobes, García-Calvo, Prieto, García-García, \& Rico-Villademoros, 2006; Davidson et al., 1997).

\section{Procedimiento y plan de análisis}

La recolección de datos fue realizada siete meses después del 27-F. Para diagnosticar el TEPT utilizamos los criterios del SPRINTE y del DTS combinadamente: diagnosticamos como TEPT cuando el criterio diagnóstico de SPRINT-E coincidió con el de DTS. Realizamos un análisis estadístico descriptivo de sus frecuencias para identificar la proporción de casos de TEPT. Finalmente, para saber si la proporción de casos fue igual o superior a la prevalencia esperada utilizamos la prueba binomial (Pardo, 2002). Supusimos que la prevalencia esperada de TEPT en la población general después del 27-F fue $22 \%$, la media entre todas las prevalencias poblacionales reportadas anteriormente (Bland et al., 2005; Cairo et al., 2010; Dewaraja \& Kawamura, 2006; Lai et al., 2004; Larrañaga \& Herrera, 2010; LeivaBianchi, 2011; McMillen et al., 2000; Roussos et al., 2005; Sharan et al., 1996; Wu et al., 2006). También utilizamos la prueba binomial para determinar si la prevalencia de un grupo fue o no superior a la del otro. Mediante el programa SPSS versión 15 realizamos los análisis de datos.

\section{RESULTADOS}

La tabla 1 muestra en detalle todas las proporciones de síntomas y prevalencia del TEPT. Resaltamos aquellas proporciones en las cuales existen diferencias importantes.

TABLA 1.

Prevalencia y porcentaje de síntomas totales y por grupos.

\begin{tabular}{|c|c|c|c|c|c|c|c|c|c|c|c|c|c|c|c|}
\hline & \multicolumn{12}{|c|}{ EPD symptoms (SPRINT-E) } & \multirow{2}{*}{\multicolumn{3}{|c|}{ Prevalencia TEPT }} \\
\hline & \multicolumn{4}{|c|}{ Síntomas TEPT } & \multicolumn{8}{|c|}{ Otras reacciones } & & & \\
\hline & $\mathrm{RE}$ & EV & EM & $\mathrm{HA}$ & $\bar{D}$ & CS & $\mathrm{TE}$ & DT & FS & $\overline{P R}$ & NA & & SPRINT-E & DTS & TEPT \\
\hline Total & 26 & 22 & 12 & 30 & 27 & 13 & 15 & 21 & 14 & 12 & 15 & 8 & $34^{* *}$ & $28^{* *}$ & 22 \\
\hline $\begin{array}{l}\text { Localización } \\
\qquad \begin{array}{r}\text { Interior } \\
\text { Costa }\end{array}\end{array}$ & $\begin{array}{c}9 \\
35^{\text {** }}\end{array}$ & $\begin{array}{c}7 \\
29^{* *}\end{array}$ & $\begin{array}{c}2 \\
17^{* *}\end{array}$ & $\begin{array}{c}13 \\
39^{* *}\end{array}$ & $\begin{array}{c}11 \\
36^{* *}\end{array}$ & $\begin{array}{c}3 \\
17^{\star *}\end{array}$ & $\begin{array}{c}6 \\
20^{* *}\end{array}$ & $\begin{array}{c}9 \\
27^{\text {** }}\end{array}$ & $\begin{array}{c}3 \\
19^{* *}\end{array}$ & $\begin{array}{c}9 \\
13^{*}\end{array}$ & $\begin{array}{c}4 \\
20^{\star *}\end{array}$ & $\begin{array}{l}6 \\
9\end{array}$ & $\begin{array}{c}14^{*} \\
44^{\star *}\end{array}$ & $\begin{array}{c}16 \\
34^{* *}\end{array}$ & $\begin{array}{l}9^{* *} \\
28^{*}\end{array}$ \\
\hline Sexo & & & & & & & & & & & & & & & \\
\hline Hombres & 18 & 8 & 8 & 16 & 18 & 7 & 10 & 14 & 10 & 10 & 10 & 11 & 21 & 15 & $8^{*}$ \\
\hline Mujeres & $29^{* *}$ & $27^{* *}$ & $14^{* *}$ & $35^{* *}$ & $31^{* *}$ & $14^{* *}$ & $17^{*}$ & $24^{* *}$ & $15^{* *}$ & 12 & $16^{* *}$ & 7 & $39 * *$ & $33^{* *}$ & 26 \\
\hline Ayuda estatal & & & & & & & & & & & & & & & \\
\hline No & 24 & 20 & 9 & 29 & 27 & 12 & 16 & 20 & 14 & 11 & 11 & 9 & $32^{* *}$ & $28 * *$ & 20 \\
\hline $\mathrm{Si}$ & 33 & $29^{*}$ & $21^{* *}$ & 34 & 30 & 14 & 14 & 25 & 13 & 13 & $25^{* *}$ & 7 & $42^{* *}$ & $29 * *$ & 25 \\
\hline Daño vivienda & & & & & & & & & & & & & & & \\
\hline Bajo & 22 & 19 & 10 & 25 & 23 & 11 & 11 & 19 & 10 & 10 & 11 & 9 & $28^{*}$ & 25 & 18 \\
\hline Alto & $39^{* *}$ & $34^{* *}$ & $21^{* *}$ & $46^{* *}$ & $42^{* *}$ & 16 & $30 * *$ & $30 *$ & $27^{* *}$ & 16 & $25^{\star *}$ & 6 & $55^{\star *}$ & $37^{* *}$ & $33^{*}$ \\
\hline Pérdida enseres & & & & & & & & & & & & & & & \\
\hline No & 25 & 17 & 9 & 29 & 24 & 13 & 10 & 20 & 12 & 12 & 13 & 9 & $30^{*}$ & 24 & 17 \\
\hline $\mathrm{Si}$ & 27 & $27^{* *}$ & $15^{*}$ & 31 & $31^{*}$ & 12 & $20^{* *}$ & 23 & 15 & 11 & 16 & 7 & $38^{* *}$ & $32^{\text {** }}$ & 25 \\
\hline
\end{tabular}

Negrita: prevalencias significativamente diferentes de 22\%; Cursiva: porcentaje del grupo que es significativamente distinto en comparación con el otro; *: $p<.05 ;$ **: $p<.01$ 
La prevalencia de TEPT para la muestra total fue de $22 \%$. El síntoma más intenso fue híper-activación $(\mathrm{HA}=30 \%)$ y el menos intenso fue el de ideación suicida (IS=8\%). Los habitantes del litoral tienen una prevalencia de $28 \%$, mientras que el $9 \%$ de quienes viven en ciudades del interior presentarían la condición. HA es el síntoma más presente entre las personas que habitan tanto en la costa (39\%) como en el interior (13\%), mientras que IS $(9 \%)$ y embotamiento $(E M=2 \%)$ son los síntomas menos intensos entre los habitantes de la costa y del interior, respectivamente.

Por sexos, $26 \%$ de las mujeres presenta TEPT. El síntoma más frecuente fue híperactivación $(\mathrm{HA}=35 \%)$ y el menos ideación suicida (IS=7\%). Por su parte, $8 \%$ de los hombres presenta este trastorno, con mayor sintomatología de re-experimentación $(\mathrm{RE}=18 \%)$ y menor en comportamientos poco saludables (CS=7\%).

Respecto de la ayuda estatal después del 27-F, quienes declararon haberla recibido presentan $25 \%$ de TEPT, mientras que quienes no la recibieron, $20 \%$. En ambos casos, el síntoma más prevalente es HA (34\% entre quienes recibieron y $29 \%$ entre quienes no recibieron ayuda) y el menos es IS (7\% entre quienes recibieron y $9 \%$ entre quienes no recibieron ayuda).

Por otro lado, quienes recibieron un alto daño en sus viviendas presentan $33 \%$ de TEPT. En cambio quienes no recibieron mayores daños presentan 18\% del trastorno. Para ambos grupos, el síntoma más frecuente es $\mathrm{HA}$ (46\% entre quienes tuvieron alto y $25 \%$ entre quienes tuvieron bajo daño) e IS, el menos ( $6 \%$ y $9 \%$ entre quienes tuvieron alto $y$ bajo daño respectivamente).

Por su parte, quienes perdieron enseres presentan $25 \%$ de TEPT, mientras que quienes no, $17 \%$. Para los dos grupos, el síntoma más prevalente es HA ( $31 \%$ entre quienes tuvieron y $29 \%$ entre quienes no tuvieron pérdidas) y el menos, IS (7\% y $9 \%$ entre quienes tuvieron y no tuvieron pérdidas respectivamente).

Realizando la prueba binomial para determinar si la proporción de casos de TEPT encontrada es mayor que la estimada, evidentemente no hallamos diferencias significativas para la proporción de TEPT estimada a partir de los casos comunes entre las escalas (ambas son iguales a $22 \%$ ). Al comparar la prevalencia de los síntomas de EPD entre los grupos utilizando el porcentaje menor como criterio, encontramos los siguientes resultados. Inicialmente, salvo la ideación suicida, todos los síntomas de TEPT y de reacciones posteriores a la catástrofe fueron significativamente mayores en la costa. Esto se confirma al observar que el $28 \%$ de prevalencia total del TEPT es significativamente mayor al $22 \%$ proyectado en el grupo de la costa, mientras que el $9 \%$ de prevalencia del grupo interior es significativamente menor que la prevalencia proyectada.

En cuanto al sexo, exceptuando la preocupación por las propias reacciones y la ideación suicida, las mujeres presentan sistemática y significativamente una proporción mayor de síntomas de EPD. Si bien esto es confirmado por las prevalencias estimadas a partir de las escalas SPRINT-E (39\%) y DTS (33\%), la estimación final del TEPT para las mujeres (26\%) no fue significativamente distinta del $22 \%$ proyectado para toda la población. Sin embargo, el hecho de que la proporción de hombres con TEPT (8\%) es significativamente menor que el $22 \%$ total, realza la mayor prevalencia del cuadro, sus síntomas y reacciones asociadas entre las mujeres.

Respecto del $25 \%$ de personas que recibieron algún tipo de ayuda estatal y fueron diagnosticadas con TEPT, esta 
proporción no es significativamente diferente de la prevalencia total estimada. Tampoco lo es el $20 \%$ de personas que nola recibieron. Sin embargo, las prevalencias pronosticadas por ambas escalas por separado indican que las personas que sí recibieron la ayuda estatal presentan niveles significativamente mayores que quiénes no. Confirma este resultado la significativamente mayor proporción de los síntomas de evitación $(E V=29 \%)$, embotamiento $(E M=21 \%)$ y necesidad de ayuda ( $\mathrm{NA}=25 \%$ ) en el grupo de personas que recibió ayuda después del $27-\mathrm{F}$ en comparación con el que no la recibió.

Por su parte, la prevalencia del TEPT entre quienes tienen mayores daños en sus viviendas $(33 \%)$ es significativamente mayor que la prevalencia esperada. Nuevamente la mayor parte de las proporciones de síntomas del TEPT y reacciones negativas asociadas evidencian lo anterior. Sólo los comportamientos poco saludables, la preocupación por las propias reacciones y la ideación suicida presentan prevalencias similares con quienes sufrieron daños severos o leves.

La prevalencia del TEPT entre las personas que perdieron enseres $(25 \%)$ no es significativamente distinta de la prevalencia pronosticada, como tampoco lo es entre quiénes no los perdieron (17\%). A pesar de esto, la estimación realizada por las escalas SPRINT-E y DTS por separado sugiere que la prevalencia del TEPT es mayor en las personas que sí perdieron enseres. Esta tendencia no queda clara al revisar la proporción de síntomas intensos al interior de la escala SPRINT-E: sólo los síntomas de evitación (EV=27\%), embotamiento $\quad(E M=15 \%)$, depresión $(D=31 \%)$ y tolerancia al estrés $(T E=20 \%)$ resultaron ser superiores al haber pérdidas de enseres en comparación con el grupo que no las tuvo.

Finalmente y como perspectiva general de las proporciones de síntomas de EPD, los síntomas más frecuentes son híperactivación ( $\mathrm{HA}=30 \%)$, depresión ( $\mathrm{D}=27 \%)$ y re-experimentación ( $\mathrm{RE}=26 \%)$; mientras que los menos frecuentes son embotamiento $(E M=12 \%)$, preocupación por las propias reacciones $(P R=12 \%)$ e ideación suicida $(I S=8 \%)$. Esta tendencia es confirmada en general por la comparación realizada de las proporciones de TEPT entre los distintos grupos.

\section{DISCUSIÓN}

Dos son los hallazgos más relevantes de esta investigación: la prevalencia del TEPT es mayor entre quienes han recibido más daños en sus hogares (33\%) y quiénes viven en la costa (28\%). Respecto del primer grupo de personas, se confirma la asociación entre la vulnerabilidad de las personas después de un desastre y el posterior surgimiento de síntomas posttraumáticos (Cougle, Resnick, \& Kilpatrick, 2009; Galea, Nandi, \& Vlahov, 2005; Goenjian et al., 2000). Quienes sufren graves daños en sus viviendas continuamente están expuestos al contexto de destrucción que significó el evento, favoreciendo la aparición de síntomas de TEPT especialmente los de reexperimentación e híper-activación. Lo mismo ocurre con los síntomas de EPD, se muestran más tristes, tienen menos cuidado por su salud (pueden alimentarse mal, no descansar lo suficiente, fumar más, incrementar el uso de alcohol y otras sustancias), sienten incomodidad con sus reacciones posteriores al evento, sienten que estas reacciones interfieren en sus actividades cotidianas (trabajo, labores del hogar 0 académicas), y sienten que les es difícil resolver problemas que antes solucionaban sin ayuda. Por otra parte, su tolerancia al estrés, funcionamiento social e ideación suicida no es diferente con quienes no han sufrido graves daños, lo cual dificultaría la labor de detección de los casos. Por lo tanto, es muy importante que los equipos de salud trabajen directamente con las personas cuyas viviendas estén más 
dañadas, independientemente de lo que observen en su funcionamiento social.

Por su parte, el grupo de personas que vivió en combinación el terremoto y el tsunami, no vivió uno, sino dos eventos impactantes. Estas personas presentan una prevalencia significativamente mayor $(28 \%)$ que el total de prevalencia (22\%), mientras que la prevalencia en los habitantes de ciudades del interior $(9 \%)$ es significativamente menor que el total. Esto se traduce en que todos los síntomas de TEPT y EPD (salvo el de ideación suicida) presentan una proporción significativamente mayor en los habitantes del litoral que en los del interior. Nuevamente, la intensidad del evento, la situación de vulnerabilidad personal y la destrucción del entorno inmediato pueden explicar estos hallazgos (Goenjian et al., 2008; Leiva-Bianchi \& Quintana, 2010). Ante eventos similares sugerimos a los equipos de salud mental concentrarse en las zonas más destruidas.

Otro hallazgo relevante es que las mujeres no poseen una prevalencia de TEPT significativamente mayor a la de la población general. Lo que ocurre es que los hombres presentan una prevalencia significativamente menor. Sin embargo, las mujeres presentan más síntomas que los hombres en todas las dimensiones, salvo en su funcionamiento social cotidiano e ideación suicida. Por lo tanto, conforman un grupo que requerirá atención después de un desastre.

Por otra parte, quienes recibieron ayuda del Estado no poseen una prevalencia significativamente distinta a la prevalencia media después de terremotos, como tampoco quienes no la recibieron. Sin embargo, la sintomatología de quienes recibieron ayuda es algo distinta: presentan más síntomas de evitación, embotamiento y sienten que no son capaces de vencer sus problemas sin asistencia. Probablemente la explicación está en la relativa similitud de esta sintomatología con la de quienes perdieron sus enseres porque, generalmente, la ayuda estatal se traduce en bienes materiales. Sugerimos a los organismos estatales afinar sus sistemas para detectar casos de personas que requieran ayuda y ampliarla al ámbito de la salud mental. Relacionado con lo anterior, la prevalencia de TEPT entre quienes perdieron (25\%) 0 no enseres (17\%) tampoco resultó ser distinta de la prevalencia total. Sin embargo, quienes perdieron enseres presentan más síntomas de evitación, embotamiento, depresión y han descuidado su salud física.

Por tanto, las campañas de ayuda posteriores a desastres debieran incluir psicoterapias efectivas para mitigar los efectos negativos en la salud mental. En ese sentido, la terapia cognitiva conductual para estrés post-desastre (Hamblen et al., 2009) adaptada a la población chilena después del 27-F (Encinas \& Valdés, 2012) podría ser de gran ayuda para estos efectos, por su comprobada efectividad en reducir síntomas de TEPT en esta población.

Finalmente, sugerimos para futuras investigaciones realizar paralelamente a las escalas aplicadas aquí, otras pruebas (p.e. PCL-C, TOP-8, entrevista clínica) para identificar los casos de TEPT. Asimismo, la aplicación de los criterios del DSM-V en próximas evaluaciones del TEPT es una oportunidad muy interesante para mejorar el diagnóstico de dicho trastorno. Al respecto, incluir evaluaciones de estados de ánimo negativos y pensamientos disociados, así como diferenciar los síntomas de TEPT con los de crisis de angustia y pánico podrían ser claves para determinar los casos de TEPT (Friedman et al., 2011; Miller et al., 2012). Estudios de comorbilidad y diagnóstico diferencial con trastornos basados en el miedo y la ansiedad (p.e. crisis de pánico), depresión y trastornos disociativos serían muy útiles en ese sentido. 
REFERENCIAS

APA-American Psychiatric Association. (2005). Diagnostic and Statistical Manual of Mental Disorders vol (4th ed. revise). Washington D.C.: American Psychiatric Association.

Bland, S., Valoroso, L., Stranges, S., Strazzullo, P., Farinaro, E., \& Trevisan, M. (2005). Long-term follow-up of psychological distress following earthquake experiences among working Italian males: a cross-sectional analysis. Journal of Nervous and Mental Disease, 193, 420-423.

Bobes, J., García-Calvo, C., Prieto, R., García-García, M., \& RicoVillademoros, F. (2006). Psychometric properties of the Spanish version of the screening scale for DSM-IV Generalized Anxiety Disorder of Carroll and Davidson. Actas Españolas de Psiquiatría, 34(2), 83-93. Retrieved from

http://www.ncbi.nlm.nih.gov/pubmed/16 552636

Bödvarsdóttir, I. \& Elklit, A. (2004). Psychological reactions in Icelandic earthquake survivors. Scandinavian Journal of Psychology, 45, 3-13.

Cairo, J. B., Dutta, S., \& Nawaz, H. (2010). The prevalence of posttraumatic stress disorder among adult earthquake survivors in Perú. Disaster Medicine and Public Health Preparedness, 4, 39-46.

Campbell, D., Felker, B., Liu, C., Yano, E., Kirchner, J., Chan, D., Rubenstein, L. \& Chaney, E. (2007). Prevalence of depression-PTSD comorbilidity: Implications for clinical practice guidelines and primary care-based interventions. Journal of General Internal Medicine, 22, 711-718.

Canetti, D., Galea, S., Hall, B., Johnson, R., Palmieri, P. \& Hobfoll, S. (2010). Exposure to prolongued socio-political conflict and the risk of PTSD and depression among Palestians. Psychiatry, 73(3), 219-231.
Cougle, J., Resnick, H., \& Kilpatrick, D. (2009). PTSD, depression, and their comorbidity in relation to suicidality: cross-sectional and prospective analyses of a national probability sample of women. Depression and Anxiety, 26, 1151-1157.

Davidson, J. R. T., Book, S. W., Colket, J. T., Tupler, L. A., Roth, S., David, D., Hertzberg, M., et al. (1997). Assessment of a new self-rating scale for posttraumatic stress disorder. Psychological Medicine, 27, 153-160.

Davidson, J., Hughes, D., Blazer, D. \& George L. (1991). Posttraumatic stress disorder in the community: an epidemiological study. Psychological Medicine, 21, 713-721.

Dewaraja, R., \& Kawamura, N. (2006). Trauma intensity and posttraumatic stress: Implications of the tsunami experience in Sri Lanka for the management of future disasters. International Congress Series, 1287, 69-73.

Eksy, A., Braun, K., Ertem-Vehid, H., Peykerli, G., Saydam, R., Toparlak, D. \& Alyanak, B. (2007). Risk factors for the development of PTSD and depression among child and adolescent victims following a 7.4 magnitude earthquake. International Journal of Psychiatry in Clinical Practice, 11(3), 190-199.

Encinas, D., \& Valdés, G. (2012). Adaptación de un procedimiento psicoterapéutico para el estrés postdesastre aplicado después del terremoto y tsunami del 27 de Febrero de 2010. Memoria de Grado, Universidad de Talca,

Friedman, M. J., Resick, P. A, Bryant, R. A, \& Brewin, C. R. (2011). Considering PTSD for DSM-5. Depression and Anxiety, 28(9), 750-69. doi:10.1002/da.20767

Galea, S., Nandi, A., \& Vlahov, D. (2005). The epidemiology of Post-Traumatic Stress Disorder after disasters. Public 
Health, 27, 78-91. doi:10.1093/epirev/mxi003

Goenjian, A. K., Steinberg, A. M., Najarian, L. M., Fairbanks, L. A., Tashjian, M., \& Pynoos, R. S. (2000). Prospective study of posttraumatic stress, anxiety, and depressive reactions after earthquake and political violence. American Journal of Psychiatry, 157(6), 911-916.

Goenjian, A., Noble, E., Walling, D., Goenjian, H., Karayan, I., Ritchie, T. \& Bailey, J. (2008). Heritabilities of symptoms of posttraumatic stress disorder, anxiety, and depression in earthquake exposed Armenian families. Psychiatric Genetics, 18, 261-266.

Hamblen, J. L., Norris, F. H., Pietruszkiewicz, S., Gibson, L. E., Naturale, A., \& Louis, C. (2009). Cognitive behavioral therapy for postdisaster distress: A community based treatment program for survivors of hurricane Katrina. Stress: The International Journal on the Biology of Stress, 206-214. doi:10.1007/s10488009-0213-3

Lai, T. J., Chang, C. M., Connor, K. M., Lee, L. C., \& Davidson, J. R. (2004). Fulland partial PTSD among earthquake survivors in rural Taiwan. Journal of Psychiatric Research, 38, 313-322.

Larrañaga, O., \& Herrera, R. (2010). Encuesta Post Terremoto: Principales resultados (p. 33). Santiago de Chile.

Leiva-Bianchi, M. C., \& Gallardo, I. (2013). Validation of the short posttraumatic stress disorder rating interview ( SPRINT-E ) in a sample of people affected by F-27 Chilean earthquake and tsunami. Anales de Psicología, 29(2), 328-334.

Leiva-Bianchi, M., \& Quintana, G. R. (2010). Factores ambientales y psicosociales vinculados a síntomas de ataque de pánico después del terremoto y tsunami del 27 de Febrero de 2010 en la zona central de Chile. Terapia Psicológica, 28(747), 161-168.
Leiva-Bianchi, M. (2011). Relevancia y prevalencia del estrés post-traumático post-terremoto como problema de salud pública en Constitución, Chile. Revista de Salud Pública, 13(4), 551559.

McMillen, J. C., North, C. S., \& Smith, E. M. (2000). What parts of PTSD are normal: Intrusion, avoidance, or arousal? Data from the Northridge, California earthquake. Journal of traumatic stress, 13(1), 57-75. doi:10.1023/A:1007768830246

Miller, M. W., Wolf, E. J., Kilpatrick, D., Resnick, H., Marx, B. P., Holowka, D. W., Keane, T. M., et al. (2012). The Prevalence and latent structure of proposed DSM-V posttraumatic stress disorder symptoms in U.S. National and Veteran Samples. Psychological Trauma: Theory, Research, Practice, and Policy. doi:10.1037/a0029730

Norris, F. H., \& Elrod, C. L. (2006). Psychosocial consequences of disaster. In Frann Norris, S. Galea, M. J. Friedman, \& P. Watson (Eds.), Methods for Disaster Mental Health Research (pp. 20-42). New York: The Guilford Press.

Norris, F., Friedman, M. \& Watson, P. (2002). 60,000 disaster victims speak: Part II. Summary and implications of the disaster mental health research. Psychiatry, 65(3), 240-260.

Norris, F., Hamblen, J., Brown, L., \& Schinka, J. (2008). Validation of the Short Posttraumatic Stress Disorder Rating Interview (expanded version, Sprint-E) as a measure of postdisaster distress and treatment need. American Journal of Disaster Medicine, 3(4), 201-212.

Norris, F., Weisshaar, D., Conrad, M., Díaz, E., Murphy, A., \& Ibañez, G. (2001). A qualitative analysis of posttraumatic stress among Mexican victims of disaster. Journal of Traumatic Stress, (14)4, 741-756.

PAHO-Panamerican Health Organization. (2010). El terremoto y el tsunami del 27 
de febrero en Chile: Crónicas y lecciones aprendidas en el sector salud [The earthquake and tsunami in Chile February 27: Chronicles and lessons learned in the health sector] (p. 111). Santiago de Chile: PAHO-Panamerican Health Organization.

Pardo, A. (2002). Análisis de datos categóricos. Madrid: UNED Ediciones.

Pérez, C., Vicente, B., Zlotnick, C., Kohn, R., Johnson, J., Valdivia, S., \& P., R. (2009). Estudio epidemiológico de sucesos traumáticos, trastorno de estrés post-traumático y otros trastornos psiquiátricos en una muestra representativa de Chile. Salud Mental, 32, 145-153.

Rodriguez, J., Zaccarelli, M., \& Perez, R. (2006). Guía práctica de salud mental en situaciones de desastres. Washington DC: PAHO-Panamerican Health Organization.

Roussos, A., Goenjian, A., Steinberg, A., Sotiropoulou, C., Kakaki, M., \& Kabakos, C. (2005). Posttraumatic stress and depressive reactions among children and adolescents after the 1999 earthquake in Ano Liosia, Greece. American Journal of Psychiatry, 162, 530-537.

Sharan, P., Chaudhary, G., Kavathekar, S., \& Saxena, S. (1996). Preliminary report of psychiatric disorders in survivors of a severe earthquake. American Journal of Psychiatry, 153, 556-558.

Shinfuku, N. (2002). Disaster mental health: Lessons learned from the Hanshin Awaji earthquake. World Psychiatry, 1, 158-159.

Solvason, H. B., Ernst, H., \& Roth, W. (2003). Predictors of response in anxiety disorders. Psychiatry: Interpersonal and Biological Processes, 26, 411-433. doi:10.1016/S0193$953 \times(03) 00027-3$

USGS-United States Geological Survey. (2013). Tsunami and earthquake research. Retrieved January $15,1 \mathrm{BC}$, from http://walrus.wr.usgs.gov/tsunami/
Wu, H.-C., Chou, P., Chou, F. H.-C., Su, C.Y., Tsai, K.-Y., Ou-Yang, W.-C., Su, T. T.-P., et al. (2006). Survey of quality of life and related risk factors for a Taiwanese village population 3 years post-earthquake. Australian and New Zealand Journal of Psychiatry, 40(4), 355-361. doi:10.1111/j.14401614.2006.01802.x.

\section{AGRADECIMIENTOS:}

Esta investigación fue realizada en el marco de los proyectos: FONDECYT No 11121330 "Construcción de un modelo predictivo del impacto psicosocial a partir de datos empíricos y simulados vinculados con el terremoto y tsunami del 27 de febrero de 2010"; Universidad de Talca, Programa para Iniciación en la Investigación (VAC 600 535)

A las psicólogas y psicólogos Loreto Álvarez, Guillermo Baher, Daniela Encinas, Mónica Fuentes, Carlos Poblete, Danae Pozo y Gabriela Valdés, por su labor en la recogida de datos para este artículo. 